\title{
Adsorption kinetic, equilibrium and thermodynamic studies of Eosin-B onto anion exchange membrane
}

\author{
Muhammad Imran Khan ${ }^{a, b, *}$, Mushtaq Hussain Lasharic, Majeda Khraisheh ${ }^{\mathrm{d}}$, \\ Shabnam Shahidae, Shagufta Zafarf, Prasert Prapamonthong, Aziz ur Rehman ${ }^{\mathrm{h}}$, \\ Saima Anjum, Naseem Akhtarf, Farzana Hanif ${ }^{\mathrm{f}}$ \\ "School of Chemistry and Material Science, University of Science and Technology of China, Hefei 230026, Anhui, China, \\ email: raoimranishaq@gmail.com (M.I. Khan) \\ ${ }^{b}$ Fujian Institute of Research on the Structure of Matter, Chinese Academy of Sciences, Fuzhou 350002, Fujian, China \\ 'Department of Zoology, The Islamia University of Bahawalpur, Bahawalpur 63100, Pakistan, email: mushtaqlashary@gmail.com \\ 'Department of Chemical Engineering, College of Engineering, Qatar University, Doha, Qatar, email: m.khraisheh@qu.edu.qa \\ ${ }^{e}$ Department of Chemistry, The University of Poonch, Rawalakot, Azad Jammu \& Kashmir, Pakistan, \\ email: shabnamshahida01@gmail.com \\ fDepartment of Chemistry, The Government Sadiq College Women University, Bahawalpur 63000, Pakistan, \\ emails:shg_zf@yahoo.com (S.ZZafar),drsaima@gscwu.edu.pk(S.Anjum),drnaseem@gscwu.edu.pk (N.Akhtar), \\ farzanakhan@yahoo.com (F. Hanif) \\ 'King Mongkut's Institute of Technology Ladkrabang, Bangkok, Thailand, email: prasert.pr@kmitl.ac.th \\ ${ }^{h}$ Department of Chemistry, The Islamia University of Bahawalpur, Bahawalpur 63100, Pakistan, email: azizypk@yahoo.com
}

Received 8 September 2018; Accepted 9 February 2019

\section{A B S T R A C T}

In this research, batch adsorption of anionic dye Eosin-B (EB) onto anion exchange membrane (AEM) (BI) from aqueous solution has been investigated at room temperature. The effect of some operating conditions such as contact time, membrane dosage, initial dye concentration and temperature on the percentage removal of EB from aqueous solution has been investigated in detail. Moreover, adsorption kinetics has been analyzed using different models such as pseudo-first-order, pseudo-second-order, Elovich, liquid film diffusion, modified Freundlich and Bangham models. Results show that adsorption data fits to the pseudo-second order kinetics very well. Non-linear isotherms containing two parameters and three parameters isotherms have been applied on experimental data. Different thermodynamic parameters such as Gibb's free energy $\left(\Delta G^{\circ}\right)$, enthalpy $\left(\Delta H^{\circ}\right)$, and entropy $\left(\Delta S^{\circ}\right)$ have been calculated, which shows that adsorption of EB onto anion exchange membrane (B1) is an exothermic process.

Keywords: Adsorption; Anion exchange membranes; Eosin-B; Kinetics; Isotherm; Thermodynamics

\footnotetext{
* Corresponding author.
} 